JRDMS Journal of Research in Dental and Maxillofacial Sciences

\title{
Comparison of Implant Stability in Sinus Lift Surgery Using Autogenous Versus Allogeneic Bone Grafts
}

\author{
M Sezavar', B Bohluli ${ }^{1}$, M Chehelamiran², S Danesh ${ }^{2}$, A Shahriar ${ }^{3}$, Z Malekpour $^{4 *}$ \\ 1-Assistant Professor, Oral and Maxillofacial Surgery Dept, Craniomaxillofacial Research Center, Dental Branch of Terhan, Islamic Azad University, \\ Tehran, Iran \\ 2-Dentist \\ 3-Oral and Maxillofacial Surgery Dept, Babol Medical University, Dental Branch, Babol, Iran \\ 4-Oral and Maxillofacial Surgery Dept, Craniomaxillofacial Research Center, Dental Branch of Terhan, Islamic Azad University, Tehran, Iran
}

\begin{tabular}{l}
\hline ARTICLE INFO \\
\hline Article Type \\
Original Article \\
\hline Article History \\
Received: Nov 2017 \\
Accepted: Dec 2017 \\
ePublished: Jan 2018
\end{tabular}

\section{Keywords:}

Bone Transplantation, Bone Substitutes, Dental Implant, Maxillary Sinus Floor Augmentation

\begin{abstract}
Background and aim: The most common method of increasing implant stability in the posterior maxilla comprises the reinforcement of bone height using bone grafts in sinus lift surgery. The purpose of the present study was to compare autogenous and allogeneic bone grafts in implant stability after open sinus lift surgery.

Materials and methods: This split-mouth clinical trial compared the implant stability in 10 patients who needed bilateral open sinus lifts, including 8 men and 2 women. Each side of each patient's jaw was assigned to either case or control groups. Open sinus lift was performed on both sides of the jaw: autogenous bone graft was used on the side considered as the control, while allogeneic bone graft was used on the side assigned to the case group. After four months, the implant stability was evaluated and

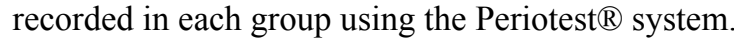

Results: The mean value related to implant stability was $-2.78 \pm 2.31$ in the control group and $-3.19 \pm 2.51$ in the case group. The values below zero (negative values) indicate an acceptable stability. According to Mann-U-Whitney test, there were no statistically significant differences between the two groups $(\mathrm{P}>0.05)$; however, the intragroup analysis using Wilcoxon test showed statistically significant results with regard to implant stability in each group $(\mathrm{P}<0.05)$.

Conclusion: Based on the results, autogenous and allogeneic bone grafts have similar effects on implant stability after open sinus lift surgery, and both bone grafts provide a suitable implant durability.
\end{abstract}




\section{Introduction:}

Posterior rehabilitation of the maxilla with dental implants is often a challenging procedure due to the weakness of the bone in this area. Loss of bone height is a consequence of alveolar bone resorption, which happens immediately after tooth extraction. ${ }^{(1)}$ The alveolar bone undergoes an additional atrophy as a result of the natural remodeling process after tooth extraction. ${ }^{(2)}$ Prosthetic rehabilitation of partially or completely edentulous maxillae without implant placement is an alternative method in severely atrophic maxillae of patients unwilling to undergo surgery. ${ }^{(3)}$ Short implants and tilted implants can be used to avoid inadvertent sinus penetration. ${ }^{(3)}$ Bone deficiency in the posterior maxilla is the most common problem encountered during treatment planning for implant-supported prostheses. ${ }^{(4)}$ In the absence of teeth, the maxillary sinus tends to pneumatized, which results in a reduced alveolar ridge height that jeopardizes the initial stability of dental implants. ${ }^{(5)}$ Elevation of the maxillary sinus floor was first introduced by Boyne and James in 1980. ${ }^{(6)}$ Sinus lifting procedures are performed to increase the height of the peri-implant bone. ${ }^{(7)}$ Maxillary sinus lifting with simultaneous dental implant insertion generally leads to an improvement in bone formation. ${ }^{(8)}$ Fresh autogenous cancellous bone grafts are currently the gold standard treatment for bone regeneration; ${ }^{(9)}$ they are the most effective agents in promoting rapid bone healing as living cells can produce bone without triggering an immune response. They also provide maximum compatibility with the host tissue and contain growth factors that stimulate new bone formation. ${ }^{(9)}$

Several attempts have been made to develop regenerative techniques that are faster compared to bone grafting procedures; however, due to the limited availability of autogenous bone and the problems associated with surgery, non-autogenous bone substitutes are still considered a treatment option. ${ }^{(10)}$ A clinical research has shown that the combination of bone grafts with other treatments can increase the speed of engraftment and formation of bone tissue as well as the healing of bone defects. ${ }^{(11)} \mathrm{A}$ suitable bone graft material should be osteoconductive, osteoinductive, and osteogenic. ${ }^{(12)}$ The allogeneic bone graft material is a bone substitute extensively used in reconstructive surgery. ${ }^{(13)}$ An allogeneic bone graft has osteoconductive properties and is similar to autologous bone; it can be used alone or in combination with other biomaterials. ${ }^{(14)}$ The main advantage of allogeneic bone grafts, compared to autogenous bone, is the exclusion of a second surgical site, which reduces patient morbidity. ${ }^{(14)}$ Accordingly, the aim of the current study was to compare the effect of autogenous and allogeneic bone grafts on implant stability after open sinus lift surgery.

\section{Materials and Methods:}

\section{Patients}

This split-mouth clinical trial involved 10 patients ( 8 men and 2 women) with the mean age of $55.8 \pm 1.77$ years (ranging from 46 to 66 years) in need of bilateral open sinus lifts and dental implants, who referred to the dental branch of Islamic Azad University of Tehran during 2016-17. The exclusion criteria comprised a history of trauma, systemic diseases, metabolic diseases, smoking, and alcohol consumption. All the patients were informed about the study protocol and signed written informed consent forms.

Study protocol

Cone-beam computed tomography (CBCT) images were taken from all patients. The minimum acceptable alveolar bone height was 4-6 mm. Each side of each patient's jaw was randomly assigned to either case or control groups. Open sinus lift was performed on both sides: autogenous bone graft was used on the side considered as the control, while allogeneic bone graft was used on the side assigned to the case group, and the implants were simultaneously inserted. An absorbable collagen membrane (Hamanand Saz Baft Kish Co., Tehran, Iran) with the thickness of 0.2-0.6 mm and the dimensions of $20 \times 30 \mathrm{~mm}$ was used. The autogenous bone graft was obtained during bone drilling for implant insertion. We used Cenobone (FDBA, 500-1000 $\mu \mathrm{m}, 1 \mathrm{cc}$, Hamanand Saz Baft Kish Co., Tehran, Iran) as an allogeneic bone graft.

All surgeries were performed by the same oral and maxillofacial surgeon. 
The outcomes were evaluated by another oral and maxillofacial surgeon, based on the radiographic images and clinical records. After four months, the stability of the implants was evaluated and recorded in each group using the Periotest $\AA$ system.

Statistical analysis

The values below zero (negative values) indicate an acceptable stability. The results were compared between the two groups using MannU-Whitney test. The intragroup analysis was performed using Wilcoxon test.

\section{Results:}

The results of implant stability after sinus lift surgery using autogenous and allogeneic bone grafts were reported in numbers between -8 to 0 , which were obtained using the Periotest ${ }^{\circledR}$ system; a greater negative number indicates a higher implant stability. The mean values related to implant stability were $-2.78 \pm 2.31$ and $-3.19 \pm 2.51$ in the control and case groups, respectively (Table 1 ).

According to Mann-U-Whitney test, there were no statistically significant differences between the two groups $(\mathrm{P}>0.05)$, indicating that both bone graft materials provided a similar implant stability. The intragroup analysis using Wilcoxon test showed statistically significant results with regard to the implant stability in each group $(\mathrm{P}<0.05)$, indicating that both bone graft materials improved the stability of the implants.

Table 1. Implant stability in sinus lift surgery using autogenous versus allogeneic bone grafts

\begin{tabular}{lcc}
\hline Groups & Stability & P-value \\
\cline { 1 - 2 } Autogenous bone graft & $-2.78 \pm 2.31$ & \\
\cline { 1 - 2 } allogeneic bone graft & $-3.19 \pm 2.51$ & $\mathrm{P}>0.05$ \\
\hline
\end{tabular}

\section{Discussion:}

The alveolar bone volume is one of the key determinants of the success of dental implant treatments. ${ }^{(15)}$ The posterior maxillary bone has challenging anatomical features, mainly due to the presence of the maxillary sinus. ${ }^{(15)}$ Sinus floor elevation has become a predictable surgical method to overcome bone height deficiencies in the posterior maxilla. ${ }^{(16)}$ This surgical technique is performed using several materials ranging from autogenous bone (the gold standard) to non-autogenous grafting materials. ${ }^{(17,18)}$

However, harvesting autogenous bone grafts increases patient morbidity and discomfort. ${ }^{(19)}$

Johansson et al studied the effect of maxillary sinus floor augmentation and simultaneous implant placement using locally harvested autogenous bone chips and debris and reported that bone grafts can be harvested at the site of the maxillary sinus augmentation procedure to enable successful loading of dental implants. ${ }^{(20)}$ Several bone substitute materials are available including human (allogeneic) and animal (xenogeneic) bone grafts as well as phylogenetic grafts and chemical materials such as hydroxyapatite. ${ }^{(21)}$ In the present study, no significant difference was observed between autogenous bone graft $(-2.78 \pm 2.31)$ and allogeneic bone graft $(-3.19 \pm 2.51)$ in implant stability. Pisoni et al studied the effect of sinus lift using autogenous bone block versus particulate autogenous grafts and reported that particulate bone grafts showed better results in sinus floor augmentation procedures. ${ }^{(3)}$ It has been reported that autogenous bone grafts are safer than allogeneic and xenogeneic bone grafts. ${ }^{(2)}$ Bone substitutes can be effectively used instead of autologous bone with optimal osteoinductive, osteoconductive, and osteogenic properties. ${ }^{(22)}$

The use of autologous bone has a $95.6 \%$ graft survival and success rate. ${ }^{(23)}$ It has been reported that autologous bone graft provides a better implant stability compared to xenogeneic, allogeneic, and synthetic bone grafting. ${ }^{(5)}$ A recent study has reported that 
pure autologous bone grafts decrease bone reconstruction after the consolidation of the grafted bone; ${ }^{(5)}$ therefore, the mixture of autologous bone and allogeneic or xenogeneic bone has been suggested to be used instead of the separate use of autologous or allogeneic grafts. ${ }^{(5)}$ Satisfactory results have been reported after the use of mixed bone grafts. (24) It seems that the synergistic activity stimulates osteogenesis as autologous bone provides osteoblasts and numerous osteogenic proteins and cytokines, while allogeneic or xenogeneic bone offers an abundant bone matrix that maintains the space during new bone generation. ${ }^{(5)}$

In use of allogeneic bone graft in maxillary reconstruction for dental implant placement, it has been reported that allogeneic bone graft can be used as an alternative to autogenous grafts in atrophic maxillary and mandibular bones. ${ }^{(6)}$ In a study, the effects of the Bio-Oss ${ }^{\circledR}$ and autogenous bone have been assessed in maxillary sinus lifting in dogs during 90 and 180 days; ${ }^{(4)}$ based on the results, the percentage of the boneimplant contact was $11.46 \%$ and $52.16 \%$ with autogenous bone and the Bio-Oss ${ }^{\circledR}$, respectively. ${ }^{(4)}$ Falah et al studied graftless sinus augmentation with simultaneous dental implant placement and reported that the consolidated bone in the augmented sinus comprised $56.7 \%$ to $59.9 \%$ vital bone tissue with a $94 \%$ overall implant survival rate. ${ }^{(7)}$ Simultaneous dental implantation with an autologous intraoral jaw bone graft is a reliable method for the reconstruction of atrophic edentulous alveolar ridges. ${ }^{(25)}$ In use of allogeneic grafts, the osteoinduction and osteoconduction phases are slower compared to the use of autogenous grafts, which is due to immunologic responses. ${ }^{(6)}$ Easy manipulation, the possibility of administration of local anesthesia, the great amount of available material, lower costs, and lower patient morbidity are the main advantages of allografts. ${ }^{(6)}$
Over the years, the use of fresh-frozen bone allografts has exponentially increased.(26) Nevertheless, in our study, autogenous and allogeneic bone grafts had similar effects on implant stability in the posterior maxilla after sinus lift surgery. Further studies are necessary to precisely determine the efficacy of autogenous and allogeneic bone grafts in the stability of dental implants.

\section{Conclusion:}

Based on the results, autogenous and allogeneic bone grafts have similar effects on implant stability after open sinus lift surgery, and both bone grafts provide a suitable implant durability.

\section{Acknowledgement:}

This article is based on general dentistry thesises No. 25042,25041 registered at the dental branch of Islamic Azad University of Tehran.

\section{References:}

1- Pozzi A, Sannino G, Barlattani A. Minimally invasive treatment of the atrophic posterior maxilla: a proof-of-concept prospective study with a follow-up of between 36 and 54 months. J Prosthet Dent. 2012 Nov;108(5):286-97.

2- Park D, Spencer JA, Koh BI, Kobayashi T, Fujisaki J, Clemens TL, et al. Endogenous bone marrow MSCs are dynamic, faterestricted participants in bone maintenance and regeneration. Cell Stem Cell. 2012 Mar 2;10(3):259-72.

3- Pisoni L, Lucchi A, Persia M, Marchi O, Ordesi P, Siervo S. Sinus lift: 3 years follow up comparing autogenous bone block versus autogenous particulated grafts. J Dent Sci. 2016 Sep;11(3):231-7.

4- Cardoso CL, Curra C, Santos PL, Rodrigues MF, Ferreira-Júnior O, de Carvalho PS. Current considerations on bone substitutes in maxillary sinus lifting. Rev Clin Periodoncia 
Implantol Rehabil Oral. 2016 Aug;9(2):1027.

5- Sbordone C, Toti P, Guidetti F, Califano L, Bufo P, Sbordone L. Volume changes of autogenous bone after sinus lifting and grafting procedures: a 6-year computerized tomographic follow-up. J Craniomaxillofac Surg. 2013 Apr;41(3):235-41.

6- Boyne PJ, James RA. Grafting of the maxillary sinus floor with autogenous marrow and bone. J Oral Surg. 1980 Aug;38(8):6136.

7- Falah M, Sohn DS, Srouji S. Graftless sinus augmentation with simultaneous dental implant placement: clinical results and biological perspectives. Int J Oral Maxillofac Surg. 2016 Sep;45(9):1147-53.

8- Altintas NY, Senel FC, Kayipmaz S, Taskesen F, Pampu AA. Comparative radiologic analyses of newly formed bone after maxillary sinus augmentation with and without bone grafting. J Oral Maxillofac Surg. 2013 Sep;71(9):1520-30.

9- Moon JW, Sohn DS, Heo JU. Histomorphometric analysis of maxillary sinus augmentation with calcium phosphate nanocrystal-coated xenograft. Implant Dent. 2015 Jun;24(3):333-7.

10- Lindhe J, Nyman S, Ericsson I, Lindhe J, Lang N, Karring T. Trauma from occlusion: Periodontal tissues. In: Lindhe J, Lang NP, Karring T. Clinical Periodontology and Implant Dentistry. Oxford: Blackwell Munksgaard, 2008:363-73.

11- Marx RE. Platelet-rich plasma: evidence to support its use. J Oral Maxillofac Surg. 2004 Apr;62(4):489-96.

12- Bauer TW, Muschler GF. Bone graft materials. An overview of the basic science. Clin Orthop Relat Res. 2000 Feb;(371):10-27.

13- De Coster P, Browaeys H, De Bruyn $\mathrm{H}$. Healing of extraction sockets filled with BoneCeramic ${ }^{\circledR}$ prior to implant placement: preliminary histological findings. Clin Implant Dent Relat Res. 2011 Mar;13(1):34-45.
14- Esposito M, Grusovin MG, Rees J, Karasoulos D, Felice P, Alissa R, et al. Effectiveness of sinus lift procedures for dental implant rehabilitation: a Cochrane systematic review. Eur J Oral Implantol. 2010 Spring;3(1):7-26. 15- Wainwright M, Torres-Lagares D, PérezDorao B, Serrera-Figallo MA, GutierrezPerez JL, Troedhan A, et al. Histological and histomorphometric study using an ultrasonic crestal sinus grafting procedure. A multicenter case study. Med Oral Patol Oral Cir Bucal. 2016 May 1;21(3):e367-73.

16- Bornstein MM, Chappuis V, von Arx T, Buser D. Performance of dental implants after staged sinus floor elevation procedures: 5 -year results of a prospective study in partially edentulous patients. Clin Oral Implants Res. 2008 Oct;19(10):1034-43.

17- Troedhan A, Kurrek A, Wainwright M. Biological principles and physiology of bone regeneration under the Schneiderian membrane after sinus lift surgery: a radiological study in 14 patients treated with the transcrestal hydrodynamic ultrasonic cavitational sinus lift (Intralift). Int J Dent. 2012;2012:576238.

18- Raja SV. Management of the posterior maxilla with sinus lift: review of techniques. J Oral Maxillofac Surg. 2009 Aug;67(8):17304.

19- Jank S, Kurrek A, Wainwright M, Bek VE, Troedhan A. Rupture length of the sinus membrane after $1.2 \mathrm{~mm}$ puncture and surgical sinus elevation: an experimental animal cadaver study. Oral Surg Oral Med Oral Pathol Oral Radiol Endod. 2011 Nov;112(5):56872.

20- Johansson LA, Isaksson S, Lindh C, Becktor JP, Sennerby L. Maxillary sinus floor augmentation and simultaneous implant placement using locally harvested autogenous bone chips and bone debris: a prospective clinical study. J Oral Maxillofac Surg. 2010 Apr;68(4):837-44.

21- Velázquez-Cayón R, Romero-Ruiz MM, Torres-Lagares D, Pérez-Dorao B, Wain- 
wright M, Abalos-Labruzzi C, et al. Hydrodynamic ultrasonic maxillary sinus lift: review of a new technique and presentation of a clinical case. Med Oral Patol Oral Cir Bucal. 2012 Mar 1;17(2):e271-5.

22- Aghaloo TL, Moy PK. Which hard tissue augmentation techniques are the most successful in furnishing bony support for implant placement? Int J Oral Maxillofac Implants. 2007;22 Suppl:49-70.

23- Sakkas A, Wilde F, Heufelder M, Winter $\mathrm{K}$, Schramm A. Autogenous bone grafts in oral implantology - is it still a "gold standard"? A consecutive review of 279 patients with 456 clinical procedures. Int J Implant Dent. 2017 Dec;3:23.

24- Beretta M, Cicciù M, Poli PP, Rancitelli D, Bassi G, Grossi GB, et al. A retrospective evaluation of 192 implants placed in augmented bone: long-term follow-up study. J Oral Implantol. 2015 Dec;41(6):669-74.

25- Pistilli R, Felice P, Piatelli M, Nisii A, Barausse C, Esposito M. Blocks of autogenous bone versus xenografts for the rehabilitation of atrophic jaws with dental implants: preliminary data from a pilot randomised controlled trial. Eur J Oral Implantol. 2014 Summer;7(2):153-71.

26- Spin-Neto R, Stavropoulos A, Dias Pereira LA, Marcantonio E Jr, Wenzel A. Fate of autologous and fresh-frozen allogeneic block bone grafts used for ridge augmentation. A CBCT-based analysis. Clin Oral Implants Res. 2013 Feb;24(2):167-73. 